\title{
GROUPS WITH AN AUTOMORPHISM CUBING MANY ELEMENTS
}

\author{
DESMOND MacHALE \\ (Received 11 February 1974; revised 1 July 1974) \\ Communicated by G. E. Wall
}

\section{Introduction}

Let $G$ be a group and $\alpha_{n}$ the mapping which takes every element of $G$ to its $n$th power, where $n$ is an integer. It is well known that if $\alpha_{n}$ is an automorphism then $G$ is Abelian in the cases $n=-1,2$, and 3. For any other integer $n(\neq 0)$ there exists a non-Abelian group which admits $\alpha_{n}$ as the identity automorphism. Indeed Miller (1929) has shown that if $n \neq 0, \pm 1,2,3$ then there exist nonAbelian groups which admit $\alpha_{n}$ as a non-trivial automorphism.

Confining our attention to finite groups, we consider the problem of how large a proportion of the elements of a non-Abelian group can be mapped to their $n$th powers by some automorphism when $n=-1,2$ or 3 . Let $\mathscr{G}_{p}$ denote the set of all finite groups with order divisible by the prime $p$ but by no smaller prime. In the case $n=-1$ it is known that if $G$ is a non-Abelian group in $\mathscr{G}_{p}$ then not more than $\frac{3}{4}|G|$ or $|G| / p$ of its elements can be inverted by an automorphism according as $p=2$ or $p$ is odd. Manning (1906) classified all groups $G$ with an automorphism inverting $\frac{3}{4}|G|$ elements, while Liebeck and the present author (1973) classified all non-Abelian groups in $\mathscr{G}_{p}$ ( $p$ odd) with an automorphism inverting $|G| / p$ elements.

Liebeck (1973) has recently settled the case $n=2$ by proving that if $G$ is a non-Abelian group in $\mathscr{G}_{p}$ then no automorphism can send more than $|G| / p$ elements of $G$ to their squares. This result includes the case $p=2$. A complete classification of all non-Abelian groups $G$ in $\mathscr{G}_{p}$ with an automorphism squaring exactly $|G| / p$ elements also appears in Liebeck (1973).

In this paper we investigate the case $n=3$. We prove the following results:

(a) If $G$ is a finite non-Abelian group then not more than $\frac{3}{4}|G|$ elements can be cubed by an automorphism.

(b) $G$ is a finite group with an automorphism cubing exactly $\frac{3}{4}|G|$ elements if and only if $G$ has central quotient group of order 4 and the centre of $G$ has no elements of order 3 . 
(c) If $G$ is a non-Abelian group in $\mathscr{G}_{p}$ and $p$ is odd then no automorphism of $G$ can send more than $|G| / p$ elements to their cubes.

\section{Notation}

$G \quad$ Denotes a finite group.

$\alpha \quad$ An automorphism of $G$.

$T \quad\left\{g \in G \mid(g) \alpha=g^{3}\right\}$.

$\mathscr{G}_{p} \quad$ The set of all groups with order divisible by the prime $p$ but by no smaller prime.

$C_{G}(t) \quad$ The centralizer of the element $t$ in the group $G$.

$\mathbf{Z}(G)=Z$ The centre of $G$.

$G^{\prime} \quad$ The commutator subgroup of $G$.

\section{Preliminary results.}

Lemma 3.1. If $\alpha \in$ Aut $G$ then $g^{-1}(g \alpha) \in C_{G}\left(T \cap g^{-1} T g\right)$.

Proof. For $g \in G, t \in T, g^{-1} t g \in T \Leftrightarrow\left(g^{-1} t g\right)^{3}=\left(g^{-1} t g\right) \alpha \Leftrightarrow\left[g^{-1}(g \alpha), t\right]=1$.

LeMmA 3.2. If $|G|$ is odd and $g \alpha=g(g \neq 1)$ then $T \cap T g$ is empty.

Proof. Suppose that $t \in T \cap T g$. Then $t=t_{1} g$ and applying $\alpha$ we get $t^{3}=t_{1}{ }^{3} g$. Thus $t^{2}=t_{1}{ }^{2}$ and the oddness of $|G|$ gives $t=t_{1}$, and $g=1$.

LEMMA 3.3. (Joseph (1969)). If $G$ is a non-Abelian group in $\mathscr{G}_{p}$ ( $p$ odd) then $G$ has at least $|G| / p$ conjugacy classes if and only if $G$ is nilpotent of class 2 with $\left|G^{\prime}\right|=p$.

Proof. $G$ has $\left(G: G^{\prime}\right)$ irreducible representations of degree 1 and hence at least $|G| / p-\left(G: G^{\prime}\right)$ other irreducible representations, each of degree at least $p$. Hence

$$
|G| \geqq|G| /\left|G^{\prime}\right|+p^{2}\left(\frac{1}{p}-\frac{1}{\left|G^{\prime}\right|}\right)|G|
$$

from which it follows that $\left|G^{\prime}\right| \leqq p+1$. Since $p$ is odd, $\left|G^{\prime}\right|=p$, and so $G^{\prime} \subseteq Z(G)$, since $G$ belongs to $\mathscr{G}_{p}$. The converse is obvious.

Lemma 3.4. If $G$ belongs to $\mathscr{G}_{p}$ and $Z(G)$ is not contained in $T$ then $|T| \leqq|G| / p$.

Proof. If $\boldsymbol{Z} \notin T$ then $T \cap \boldsymbol{Z}$ is a proper subgroup of $\boldsymbol{Z}$. Clearly, $|Z x \cap T| \leqq(1 / p)|Z|$ for any $x$ in $G$ and the result follows. 


\section{Main Results}

Theorem 4.1. If $|T|>\frac{3}{4}|G|$ then $T=G$ and $G$ is Abelian.

Proof. Suppose that $|T|>\frac{3}{4}|G|$ and let $t$ be any element of $T$. Then

$$
\left|t^{-1} T t \cap T\right|=\left|t^{-1} T t\right|+|T|-\left|t^{-1} T t \cup T\right|>\frac{3}{4}|G|+\frac{3}{4}|G|-|G|=\frac{1}{2}|G| \text {. }
$$

By Lemma 3.1, $t^{2}$ commutes with more than half the elements of $G$ and hence $t^{2} \in Z(G)$, for all $t \in T$.

Similarly, $|t T \cap T|>\frac{1}{2}|G|$. However, if $t, s$ and $t s$ belong to $T$ then $t^{3} s^{3}=(t s)^{3}$ and so $t s=s t$, since $t^{2}$ is central.

Hence, $\left|C_{G}(t)\right|>\frac{1}{2}|G|$ for every $t \in T$ and so every element of $T$ is central. Finally, $|Z(G)|>\frac{3}{4}|G|$ and so $T=Z(G)=G$.

THEOREM 4.2. $G$ has an automorphism for which $|T|=\frac{3}{4}|G|$ if and only if $(G: Z(G))=4$ and $Z(G)$ has no elements of order 3 .

Proof. If $(G: Z(G))=4$ then $G=\boldsymbol{Z} \cup \boldsymbol{Z} a \cup \boldsymbol{Z} b \cup \boldsymbol{Z} a b$ where $a^{2}, b^{2}$ and $[a, b]$ all belong to $\boldsymbol{Z}$. A routine calculation shows that if $\boldsymbol{Z}$ has no elements of order 3 then the map defined by $z a^{i} b^{j} \rightarrow z^{3} a^{3 i} b^{3 j}, 0 \leqq i, j \leqq 1$ for all $z \in Z$, defines an automorphism sending exactly $\frac{3}{4}|G|$ elements to their cubes.

Conversely, let $G$ be a group for which $|T|=\frac{3}{4}|G|$. Clearly $G$ is non-Abelian. Let $t$ be any non-central element of $T$. We show that $C_{G}(t)$ is an Abelian subgroup of index 2 in $G$.

As in the proof of Theorem $4.1\left|C_{G}\left(t^{2}\right)\right| \geqq \frac{1}{2}|G|$. If $\left|C_{G}\left(t^{2}\right)\right|>\frac{1}{2}|G|$ then $t^{2}$ is central and thus $|t T \cap T| \geqq \frac{1}{2}|G|$. Thus $\left|C_{G}(t)\right| \geqq \frac{1}{2}|G|$ and since $t$ central, $\left|C_{G}(t)\right|=\frac{1}{2}|G|$. Moreover, $C_{G}(t) \subset T$ and so $C_{G}(t)$ is Abelian.

We can now assume that $\left|C_{G}\left(t^{2}\right)\right|=\frac{1}{2}|G|$ and $C_{G}\left(t^{2}\right)$ is Abelian, since $C_{G}\left(t^{2}\right) \subset T$. Accordingly, if $g t^{2}=t^{2} g$ then $g t=t g$ since $t t^{2}=t^{2} t$. So $C_{G}(t)=$ $C_{G}\left(t^{2}\right)$ and $C_{G}(t)$ is an Abelian subgroup of index 2 in $G$.

Finally, let $a$ and $b$ be a pair of non-commuting elements of $T$. Such a pair exists since otherwise $G$ is Abelian. Let $A=C_{G}(a)$ and $B=C_{G}(b)$ and so $A$ and $B$ are distinct Abelian subgroups of index 2 in $G$.

Now $G=A B$ and $(G: A \cap B)=4$. Clearly $A \cap B=Z(G)$. Since $Z(G) \subset T$, $\boldsymbol{Z}(G)$ has no elements of order 3 and the proof is complete.

THEOREM 4.3. Let $G \in \mathscr{G}_{p}$ and let $G$ be non-Abelian, where $p$ is odd. Then $|T| \leqq|G| / p$, for any automorphism $\alpha$ of $G$.

Proor. Suppose that $G \in \mathscr{G}_{p}$ and $|T|>(1 / p)|G|$, where $G$ is non-Abelian. We first consider the case where $\alpha$ fixes a non-trivial element $g$ of $G$. Now $g$ has order at least $p$ and by Lemma 3.2 the $p$ sets $T, T g, \cdots, T g^{p-1}$ are pairwise disjoint. Then, $|G| \geqq\left|T \cup T g \cup \cdots \cup T g^{p-1}\right|=p|T|>|G|$, a contradiction. 
We may thus assume that $\alpha$ is fixed-point-free. By Lemma 3.1, for $g \in G$, $t \in T, g^{-1} t g \in T$ if and only if $\left[g^{-1}(g \alpha), t\right]=1$. Since $\alpha$ is fixed-point-free the correspondence $g^{-1} g(\alpha) \leftrightarrow g$ is one-to-one and so $g^{-1} t g \in T \Leftrightarrow[g, t]=1$. Hence any conjugacy class contains at most one element of $T$. Thus $G$ has at least $(1 / p)|G|$ conjugacy classes and so by Lemma 3.3, $G$ is nilpotent of class 2 with $\left|G^{\prime}\right|=p$. Moreover, by Lemma 3.4, $Z(G) \subset T$ and so $G^{\prime} \subseteq Z(G) \subset T$.

Finally, let $r$ and $s$ be a pair of noncommuting elements of $T$. Then, $[r, s] \alpha=\left[r^{3}, s^{3}\right]=[r, s]^{3}=[r, s]^{9}$, since $r, s \in T$ and $G$ is nilpotent of class 2 . Thus $[r, s]^{6}=1$ and so $[r, s]^{3}=1$, by the oddness of $|G|$. Since $T$ has no elements of order 3 , this is a contradiction and the theorem is established.

\section{References}

K. S. Joseph (1969), Commutativity in non-Abelian groups (Ph. D. thesis, University of California, Los Angeles, 1969).

H. Liebeck (1973), 'Groups with an automorphism squaring many elements', J. Austral. Math. Soc. 16, 33-42.

H. Liebeck and D. MacHale (1973), 'Groups of odd order with automorphisms inverting many elements', J. London Math. Soc. (2) 6, 215-223.

W. A. Manning (1906), 'Groups in which a large number of operators may correspond to their inverses', Trans. Amer. Math. Soc. 7, 233-240.

G. A. Miller (1929), 'Possible $\alpha$-automorphisms of non-Abelian groups', Proc. Nat. Acad. Sci. 15, 89-91.

Department of Mathematics

University College

Cork

Ireland. 